\title{
Diabetes and bone fracture: risk factors for old and young
}

\author{
Peter Vestergaard
}

Received: 6 May 2014 / Accepted: 7 July 2014 / Published online: 27 July 2014

(C) Springer-Verlag Berlin Heidelberg 2014

\begin{abstract}
Diabetes mellitus may affect bone turnover in a number of ways, thus leading to impaired bone quality and a consequent increase in fracture risk. These factors are summarised in this commentary.
\end{abstract}

Keywords Bone mineral density · Diabetes mellitus · Fracture - Osteocalcin - Type 1 diabetes - Type 2 diabetes

\section{Abbreviation \\ BMD Bone mineral density}

Diabetes and the skeleton have been linked on a number of levels, including an increased risk of fractures. Diabetes may affect both the young and the elderly. The mechanisms behind the increased fracture risk may be related to:

1. Low-grade inflammation mainly linked to obesity affecting turnover in the skeleton [1]. This would predominantly be expected to be a factor in type 2 diabetes. It would by nature lead to a low turnover state in the bones, which is what is seen in diabetes [2, 3].

2. An altered relationship between the beta cells and bone [4], as osteocalcin is involved in bone turnover but may also affect the beta cells [5]. If bone turnover is low, osteocalcin levels will decrease, and this may in theory further impair insulin secretion from the beta cells.

P. Vestergaard $(\bowtie)$

Department of Clinical Medicine, Aalborg University Hospital and Aalborg University, Mølleparkvej 4, DK-9100 Aalborg, Denmark e-mail: p-vest@post4.tele.dk

\section{P. Vestergaard}

Department of Endocrinology, Aalborg University Hospital and

Aalborg University, Aalborg, Denmark
3. Decreased bone turnover resulting from decreased osteoclastic resorption in bone with glycated collagen [2, 3]. Because of the coupling between osteoclasts and osteoblasts, bone turnover would decrease.

4. Altered glycation of collagen and thus impaired biomechanical competence [6]. An increase in fractures stemming from this mechanism would not necessarily be reflected in changes in areal bone mineral density (BMD) determined by dual-energy X-ray absorptiometry (DXA) or other scanning techniques relying on measurements of calcium content of the bones.

5. Lack of the anabolic effects of insulin, which would be present in type 1 diabetes, and less so in type 2 diabetes, where insulin levels may be increased. However, altered levels of IGF1 may also play a role [7].

6. Negative calcium balance brought about by hyperglycaemia through increased urinary calcium excretion [8] and altered intestinal calcium absorption [9].

7. Alterations in vitamin D metabolism [10] — in particular in patients with renal disease.

8. Functional hypoparathyroidism [11]. This may be a less significant factor, as, in theory, hypoparathyroidism should lead to increased [12] and not decreased BMD.

9. Drug-specific effects, such as negative effects of peroxisome proliferator-activated receptor $\gamma$ agonists [13], but also potential effects of other drugs, such as insulin or the oral glucose-lowering drugs.

Besides these mechanisms, which are linked to bone biomechanical competence, increased risk of falls (for example, from peripheral neuropathy, impaired vision, hypoglycaemia, ischaemic cerebral events) may also play a role. These effects would not be linked to BMD.

To further study these factors it is necessary to determine whether differences exist with age (say, older individuals being more susceptible than younger) or whether sex 
differences are present. Sex-specific effects could, for example, be related to oestrogen or aromatase activity in women. If older individuals with diabetes were more susceptible, this could point to factors associated with age, such as alterations in bone architecture or the ratio of collagen to hydroxyapatite. Sex-specific differences could point to effects linked to aromatase or to testosterone or oestrogen.

Regarding differences between type 1 diabetes and type 2 diabetes, it has been reported that although an increased risk of fractures may be seen in both conditions [14, 15], BMD seems to be increased in type 2 diabetes, but this does not confer protection against fractures, as would otherwise be expected. As reported in this issue of Diabetologia, in their study adjusting for BMD, Napoli et al observed an increased risk of fractures, i.e. a risk beyond what can be explained by BMD, among the subgroup of older men with diabetes vs those with normoglycaemia [16]. No clear distinction between type 1 and type 2 diabetes is made in the paper, but the majority of individuals may be expected to have type 2 diabetes. Like Strotmeyer et al [17], the study by Napoli et al did not find a relationship with impaired fasting glucose. However, Napoli et al did report a relationship with the use of insulin. Insulin may counter hyperglycaemia and thus counter some of the negative effects on bone. However, lack of sufficient intrinsic insulin production may also signal a lack of the potential anabolic effects of insulin, which may not be mimicked by addition of external insulin.

The patients in the study were predominantly elderly type 2 diabetic patients. Insulin treatment in these patients would often be an add-on to, for example, oral glucose-lowering drugs and could signal longstanding and perhaps poorly controlled diabetes. Poorly controlled diabetes needing addition of insulin could thus have led to impaired integrity of the skeleton, as outlined above. Alternative explanations may be increased risk of falls linked to, for example, complications with peripheral neuropathy or affection of the central nervous system linked to, say, ischaemia or stroke.

In conclusion, diabetes may-beyond the well-known acute and late complications - be a risk factor for skeletal frailty in both young and old alike. The possible mechanisms involved span from signalling between the skeleton and beta cell to negative effects on the bone competence by hyperglycaemia.

Duality of interest The author declares that there is no duality of interest associated with this manuscript.
Contribution statement The author was the sole contributor to this paper.

\section{References}

1. Donath MY, Shoelson SE (2011) Type 2 diabetes as an inflammatory disease. Nat Rev Immunol 11:98-107

2. Valcourt U, Merle B, Gineyts E et al (2007) Non-enzymatic glycation of bone collagen modifies osteoclastic activity and differentiation. J Biol Chem 282:5691-5703

3. Jiajue R, Jiang Y, Wang O et al (2014) Suppressed bone turnover was associated with increased osteoporotic fracture risks in non-obese postmenopausal Chinese women with type 2 diabetes mellitus. Osteoporos Int. 25:1999-2005

4. Lee N, Sowa H, Hinoi E et al (2007) Endocrine regulation of energy metabolism by the skeleton. Cell 130:456-469

5. Kindblom JM, Ohlsson C, Ljunggren O et al (2009) Plasma osteocalcin is inversely related to fat mass and plasma glucose in elderly Swedish men. J Bone Miner Res 24:867-874

6. Saito M, Marumo K (2010) Collagen cross-links as a determinant of bone quality: a possible explanation for bone fragility in aging, osteoporosis, and diabetes mellitus. Osteoporos Int 21:195-214

7. Jehle PM, Jehle DR, Mohan S et al (1998) Serum levels of insulin-like growth factor system components and relationship to bone metabolism in type 1 and type 2 diabetes mellitus patients. J Endocrinol 159:297-306

8. McNair P, Madsbad S, Christensen M et al (1979) Bone mineral loss in insulin-treated diabetes mellitus: studies on pathogenesis. Acta Endocrinol 90:463-472

9. Carnevale V, Romagnoli E, D'Erasmo E (2004) Skeletal involvement in patients with diabetes mellitus. Diabetes Metab Res Rev 20:196-204

10. Bouillon R (1991) Diabetic bone disease [editorial]. Calcif Tissue Int 49:155-160

11. McNair P, Christensen M, Madsbad S et al (1981) Hypoparathyroidism in diabetes mellitus. Acta Endocrinol (Copenh) 96:81-86

12. Abugassa S, Nordenstrom J, Eriksson S et al (1993) Bone mineral density in patients with chronic hypoparathyroidism. J Clin Endocrinol Metab 76:1617-1621

13. Benvenuti S, Cellai I, Luciani P et al (2007) Rosiglitazone stimulates adipogenesis and decreases osteoblastogenesis in human mesenchymal stem cells. J Endocrinol Invest 30:RC26-RC30

14. Janghorbani M, van Dam R, Willett W et al (2007) Systematic review of type 1 and type 2 diabetes mellitus and risk of fracture. Am J Epidemiol 166:495-505

15. Vestergaard P (2007) Discrepancies in bone mineral density and fracture risk in patients with type 1 and type 2 diabetes - a metaanalysis. Osteoporos Int 18:427-444

16. Napoli N, Strotmeyer ES, Ensrud KE et al (2014) Fracture risk in diabetic elderly men: the MrOS study. Diabetologia. doi:10.1007/ s00125-014-3289-6

17. Strotmeyer E, Cauley J, Schwartz A et al (2005) Nontraumatic fracture risk with diabetes mellitus and impaired fasting glucose in older white and black adults: the health, aging, and body composition study. Arch Intern Med 165:1612-1617 